\title{
La Red de Restauración Ecológica de la Argentina (REA): Avances, vacíos y rumbo a seguir
}

\author{
Daniel Pérez ${ }^{1, \otimes}$; Paula Meli ${ }^{2 / 3} ;$ Daniel Renison $^{4} ;$ Fernando Barri $^{5}$; Adriana \\ Beider ${ }^{6}$; Gabriel Burgueño ${ }^{7}$; Antonio Dalmasso; ${ }^{;}$Sebastián Dardanelli ${ }^{9} ;$ \\ Manuel de Paz ${ }^{10}$; Fernando Farinaccio'; Gabriela Papazian ${ }^{12}$; Martín \\ SIROMBRA ${ }^{13} \&$ ROMINA TORRES ${ }^{4}$
}

\begin{abstract}
${ }^{1}$ Laboratorio de Rehabilitación y Restauración de Ecosistemas Áridos y Semiáridos (LARREA). Facultad Ciencias del Ambiente y la Salud (FACIAS). Universidad Nacional del Comahue. Neuquén. Argentina. ${ }^{2}$ Departamento de Ciencias Forestales, Escuela Superior de Agricultura "Luiz de Queiroz", Universidad de São Paulo, Brasil. ${ }^{3}$ Fundación Internacional para la Restauración de Ecosistemas, España. ${ }^{4}$ Instituto de Investigaciones Biológicas y Tecnológicas, Centro de Ecología y Recursos Naturales Renovables (CONICET - Universidad Nacional de Córdoba). Argentina. ${ }^{5}$ Instituto de Diversidad y Ecología Animal (IDEA), CONICET-UNC. Argentina. ${ }^{6}$ Instituto Nacional de Tecnología Agropecuaria (INTA), Chubut. Argentina. Laboratorio Ecotono, INIBIOMA (CONICET - Universidad Nacional del Comahue). ${ }^{7}$ Cuerpo Municipal de Guardaparques. Reserva Municipal Los Robles. Moreno. Buenos Aires, Argentina. ${ }^{8}$ Grupo de Geobotánica y Fitogeografía - IADIZA - CONICET. Argentina. ${ }^{9}$ Instituto Nacional de Tecnología Agropecuaria (INTA), EEA Paraná. Entre Ríos, Argentina. ${ }^{10}$ Laboratorio Ecotono, INIBIOMA (CONICET - Universidad Nacional del Comahue). ${ }^{12}$ Facultad de Ciencias Naturales y Ciencias de la Salud, Universidad Nacional de la Patagonia San Juan Bosco. Esquel, Chubut, Argentina. ${ }^{13}$ Cátedra Ecología General, Instituto de Limnología del Noroeste Argentino, Facultad de Ciencias Naturales e Instituto Miguel Lillo. Universidad Nacional de Tucumán. Tucumán. Argentina.
\end{abstract}

\begin{abstract}
Resumen. En un contexto latinoamericano y nacional de degradación ambiental creciente, describimos la percepción de los miembros de la REA sobre el estado actual de la restauración en la Argentina e identificamos el panorama de avances y logros a fin de sugerir acciones de restauración ecológica. La REA se formalizó en 2012 y realizó su primer encuentro nacional organizativo en 2017. Al presente, cuenta con 198 miembros distribuidos en ocho nodos regionales. Los análisis efectuados en este encuentro muestran relativamente pocas iniciativas de restauración a nivel institucional y a escala de paisaje. La mayoría de las acciones son realizadas a escala local por científicos que aportan la investigación y gestores que promueven la actividad en un rango amplio de contextos sociales y ecológicos. Los practicantes de la restauración (i.e., quienes ejecutan los proyectos a campo) usan la información que generan los sectores científico, social, privado y de gobierno. Entre otras deficiencias, mencionan la baja disponibilidad de información ecológica de base y la inexistencia o insuficiencia de marcos legales y capacidades técnicas e institucionales. Esperamos que la consolidación de la REA le permita a todos los interesados e involucrados en la restauración ecológica del país compartir experiencias y generar vínculos para aportar a la continuidad de un trabajo multidisciplinario, participativo, integrado a la problemática socioambiental del país, a diferentes escalas y con una perspectiva a largo plazo.
\end{abstract}

[Palabras clave: actores sociales, degradación, marcos legales, percepciones sociales, redes]

\begin{abstract}
The Argentine Network of Ecological Restoration (REA): Progress, gaps and future pathway. In a Latin American and national context of increasing environmental degradation, we describe the perception of the members of the REA on the current state of restoration in Argentina, and we identify the current prospect of progress and achievements in order to suggest ecological restoration actions in the country. The REA was formalized in 2012 and held its first national organizational meeting in 2017. It currently has 198 members distributed in eight regional nodes. Analyses carried out at this meeting indicate relatively few restoration initiatives at the institutional and landscape levels. Most of the actions are carried out at the local level by scientists who contribute through research and practitioners who promote the activity in a wide range of social and ecological contexts. Restoration practitioners (i.e., those who execute projects in the field) use information generated by the scientific, social, private and government sectors. Among other deficiencies, they mention the limited availability of basic ecological information, the inexistence or inadequacy of legal frameworks, institutional and technical capacities. We hope the consolidation of the REA will allow all those interested and involved in the ecological restoration of the country to share experiences and generate links to contribute to the continuity of a multidisciplinary, participatory work, integrated to the socio-environmental problems of the country, at different scales and with a long-term perspective.
\end{abstract}

[Keywords: degradation, legal frameworks, networks, social perceptions, stakeholders]

Editor asociado: Esteban Jobbágy

$\triangle$ danielrneuquen@gmail.com
Recibido: 2 de octubre de 2017

Aceptado: 8 de febrero de 2018 


\section{INTRODUCCIÓN}

La Argentina y el resto de Latinoamérica sufren tasas de degradación ambiental alarmantes que ponen en riesgo el desarrollo humano (Díaz et al. 2018). Tasas de deforestación muy superiores a la media global, cientos de miles de hectáreas desertificadas por año y otras grandes problemáticas ambientales requieren no sólo cambiar el rumbo del modelo económico, sino también asegurar una ganancia neta en la extensión y la funcionalidad de los ecosistemas nativos mediante inversiones en actividades de reparación ambiental, incluida la restauración ecológica (McDonald et al. 2016; Ceccon and Pérez 2017). La restauración ecológica es el proceso de ayudar al restablecimiento de un ecosistema que fue degradado, dañado o destruido, para favorecer su recuperación con respecto a su salud, integridad y sustentabilidad (SER 2004). Este proceso incluye múltiples objetivos como alcanzar y compartir beneficios socioeconómicos, suministrar servicios ecosistémicos como la provisión de agua limpia y mejorar el hábitat de especies claves para el ecosistema, lo que considera el bienestar de las comunidades humanas que sostienen y son sostenidas por los ecosistemas restaurados (Armesto et al. 2007; Higgs et al. 2014).

Una gran parte de los proyectos de restauración tiene el potencial de proveer oportunidades de participación social múltiples y permanentes (Gross 2006). En los proyectos de restauración ecológica se pueden consensuar el diseño y los objetivos entre actores heterogéneos de la sociedad, a la vez que es posible manejar las situaciones diversas que ocurren en la práctica $y$, si es necesario, volver a rediseñar las estrategias de intervención. En Latinoamérica, la necesidad de muchos practicantes e investigadores de integrarse y realizar una contribución conjunta más relevante y significativa que las acciones individuales para resolver los desafíos de la práctica hicieron surgir redes de colaboración tanto a nivel nacional (Aguilar et al. 2015; Ceccon et al. 2015; Echeverría et al. 2015) como a escalas locales y regionales (Ceccon and Pérez 2017). Entre ellas figuran la Red Brasilera de Restauración Ecológica (REBRE), la Red Colombiana de Restauración (REDCRE), la Red Mexicana de Restauración Ambiental (REPARA), la Red Chilena de Restauración Ecológica (Restauremos Chile), la Red Venezolana de Restauración Ecológica y la Red Ecuatoriana de Restauración del Paisaje.

La acción ambiental, entendida como el esfuerzo de un conjunto de actores (gubernamentales, no gubernamentales y comunitarios) para mantener los valores biológicos y culturales asociados a lugares específicos, usa con frecuencia, en el ámbito latinoamericano, la metáfora de "red" para referirse a la construcción de alianzas que buscan aunar esfuerzos para obtener un impacto mayor (Leff 2009). La participación amplia que caracteriza a este tipo de organización hace posible compartir conocimientos y experiencias, generar proyectos conjuntos y construir progresivamente nuevos vínculos entre la sociedad y la naturaleza (Jordan 2012; Cisneros and Cisneros 2016). En este contexto ocurren una serie de hitos que llevan a la formación de la Red de Restauración Ecológica de Argentina (REA). En el año 2012 se formalizó la REA en la Reunión Anual de la Asociación Argentina de Ecología (AsAE) y se eligió el primer coordinador. En 2014 se comenzaron a discutir los elementos básicos necesarios para construir un plan nacional de restauración, así como también las áreas prioritarias a restaurar, y se estableció la organización de la red con nodos que constituyen grupos que se organizan en las diferentes regiones del país para promover y desarrollar acciones de restauración y difundir la problemática de la degradación de los ecosistemas (Zuleta et al. 2015). En 2017 se realizó el primer encuentro nacional en el que se compartieron diagnósticos y resultados sobre el estado de la restauración ecológica en las regiones que cubren los nodos, y se sistematizaron percepciones sobre diversos aspectos científicos y sociales del tema. Ochenta personas participaron del evento. Entre ellas figuraron investigadores, practicantes y educadores de los sectores público y privado, quienes dan presencia de la red en 15 provincias del país. Se efectuaron compromisos y acuerdos que fueron definiendo la organización y estructura de la red, la comunicación interna entre miembros y la difusión de las acciones de restauración.

La ciencia y la práctica de la restauración ecológica en la Argentina creció de forma notable en los últimos años. Sin embargo, aún es posible detectar algunos vacíos de información acerca de ciertas ecorregiones del país y también de determinados aspectos (e.g., sociales o económicos) (Rovere 2015). Aquí, nuestro objetivo es abordar las capacidades 
y vacíos actuales para desarrollar iniciativas de restauración ecológica en la Argentina, determinar el estado actual del marco legal, los principales proyectos en desarrollo y los pasos a seguir para la consolidación de la restauración ecológica en el país. Como REA, nos hicimos las siguientes preguntas: ¿Qué tenemos? ¿Qué falta? ¿Hacia dónde vamos? Nuestro trabajo no pretende ser una revisión de la situación actual de la disciplina en la Argentina, sino brindar la percepción de un grupo de actores que tienen experiencia de largo plazo en este tema y en el país. Consideramos que las respuestas a las preguntas que nos planteamos representarán un aporte al establecimiento de una línea de base para el debate acerca del desarrollo y el fortalecimiento de las imperiosas acciones de restauración ecológica necesarias en nuestro país, con una perspectiva que abarque diferentes escalas espaciales y en el largo plazo.

\section{LA PERCEPCIÓN DE LA REA SOBRE EL ESTADO ACTUAL DE LA RESTAURACIÓN ECOLÓGICA EN LA Argentina}

\section{¿Qué tenemos?}

Se obtuvo un primer relevamiento de proyectos que fueron considerados de gran aporte en los nodos por su innovación, extensión o tiempo de ejecución, que constituyó en sí un diagnóstico para generar una futura base de datos sobre acciones de restauración en el país. Si bien la información recopilada es muy útil para obtener un panorama de la situación actual, consideramos que el producto más importante alcanzado fue la reflexión sobre facilitadores y obstáculos que estos proyectos tuvieron en su implementación (Tabla S1). Por otra parte, observamos que las acciones de restauración se implementan desde una visión interdisciplinaria, en muchos casos desde el enfoque de socio-ecosistemas, y no sólo en su componente ecológico. Es decir, los aspectos sociales y legales están siendo incluidos (e.g., la problemática institucional de la degradación de tierras). En su gran mayoría, las acciones de restauración ecológica son de escala local, desarrollados por sectores académicos y con fuerte presencia de voluntarios. Aún son escasas las iniciativas de restauraciones a nivel institucional y a escala de paisaje (e.g., corredores biológicos) que incluyan estrategias, indicadores y monitoreo a largo plazo.
En cuestiones de normativa legal, la implementación de restauración en la Argentina se encuadra en el artículo 41 de la Constitución Nacional, que establece que el daño ambiental generará prioritariamente la obligación de "recomponer", según lo establezca la Ley. Actualmente, a escala nacional se cuenta con la Ley General del Ambiente (Ley 25.675) y con otras nacionales o provinciales que mencionan la palabra restauración, rehabilitación, repoblamiento, recuperación, recomposición o manejo (Tabla S2). Si bien las normas no utilizan definiciones reconocidas por la academia sobre restauración ecológica, las mismas promueven marcos institucionales desde los cuales se pueden fundamentar acciones de restauración como la entienden los restauradores (SER 2004; McDonald et al. 2016). Se observa que las normas que más énfasis hacen en la restauración alcanzan a actividades como la minería y la extracción forestal y de hidrocarburos, pero, a la vez, se omiten en otras actividades degradantes como la agricultura y la ganadería. En general, los instrumentos jurídicos son muy heterogéneos en exigencias para efectuar las acciones de restauración entre las diferentes provincias. La heterogeneidad también se extiende hacia el resto de Latinoamérica, ya que si bien hoy en día parece impensable la restauración de campos agrícolas de soja y otros granos, es una realidad en países como Brasil, que cuenta con un código forestal que limita, reglamenta y establece exigencias de restauración en la agricultura (Soares-Filho et al. 2014; Brancalion et al. 2016). Algunas leyes mencionadas en la Tabla S2 no cuentan con financiamiento o no definen sus alcances ni tipos de acciones a ejecutar, lo que se traduce en que sean muy pocos los casos de desmontes ilegales que hayan sido restaurados (Vera et al. 2017). Las definiciones vagas y la falta de regulación y control por parte de las autoridades de aplicación se prestan para que ocurran ambigüedades en la aplicación; además, no existen monitoreos de implementación. Este problema no es exclusivo de la Argentina, ya que en Latinoamérica sólo Brasil logró instrumentos legales eficaces para la ejecución de sus planes de restauración (Méndez-Toribio et al. 2017).

En cuanto a lo académico, los miembros de la REA opinan que la investigación vinculada con la restauración ecológica en la Argentina se está abordando de forma sostenida y en varias escalas ecológicas (e.g., poblaciones, 

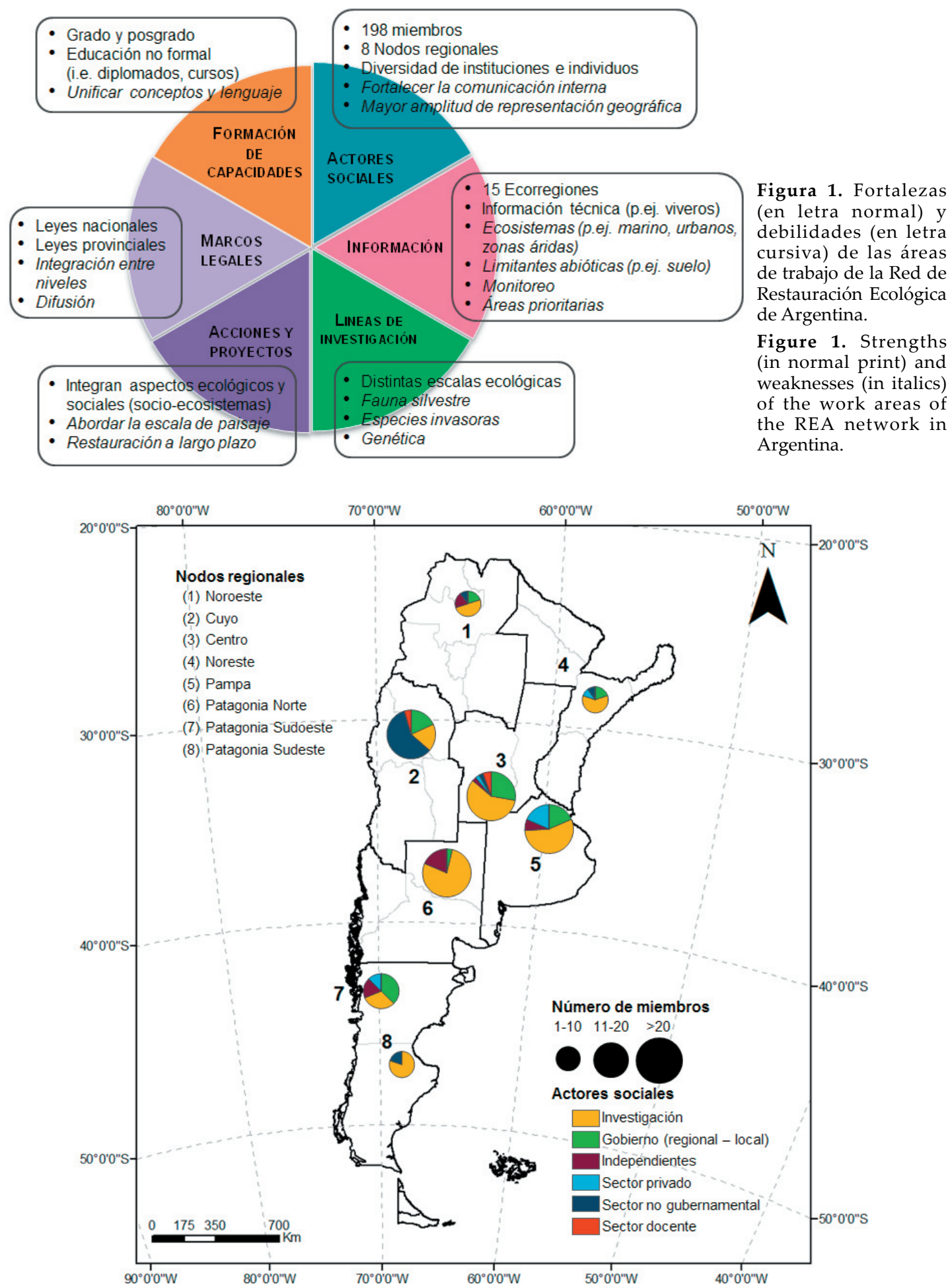

Figura 2. Conformación actual de la REA. Área del país que abarca cada uno de los nodos regionales y distribución de miembros y actores sociales. El tamaño de los círculos es proporcional al número de miembros en cada nodo (distribución de miembros). Dentro de cada círculo se muestra la proporción de los distintos tipos de actores sociales en cada nodo (distribución de actores sociales; ver Figura 3).

Figure 2. Current configuration of the REA. Area of the country that includes each of the regional nodes and distribution of members and social actors. The size of the circles indicates the number of members in each node (distribution of members). Within each circle, the proportion of different types of social actors in each node is shown (distribution of social actors, see Figure 3). 
comunidades), aunque existe un déficit importante de investigaciones en manejo de especies exóticas y en restauración de fauna (Figura 1). En relación con la oferta de formación en restauración tanto a nivel de grado como de posgrado, el panorama es muy heterogéneo y con grandes diferencias en cada región del país. Según relevamientos de la REA, existe un doctorado que incluye extensamente la restauración, cinco maestrías y tres especializaciones, nueve cursos dentro de formaciones de posgrado y cinco en formaciones de grado, dos experiencias de educación no formal, una técnica y una de formación docente (Tabla S3). Sin embargo, todavía se considera necesario y relevante unificar los conceptos y el lenguaje utilizado, ya que muchas asignaturas y contenidos se vinculan sólo de forma indirecta con el marco de restauración que en la actualidad acepta la comunidad académica. Asimismo, se necesita una labor fuerte a fin de incorporar la restauración ecológica a los currículos de grado y pre-grado, como así también para aumentar las ofertas de formación (tanto formales como no formales) en todos los niveles de la educación y en la población en general. En este panorama general, consideramos que la REA puede generar un espacio para avanzar en las dificultades que se presentan.

La organización de la REA tiene ciertas fortalezas y algunas debilidades que resumimos en la Figura 1. Actualmente, la red está conformada por 198 miembros, quienes participan en ocho nodos regionales (Figura 2). Estos miembros representan a una gran diversidad de actores sociales (Figura 3). Si bien la mayor parte de sus miembros provienen del área de la investigación (60\%, universidades y centros de investigación), también están presentes las agencias de

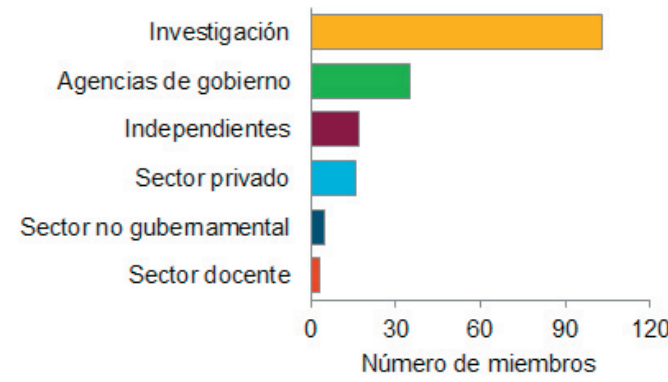

Figura 3. Representación de diversos tipos de actores sociales entre los miembros actuales de la red REA a escala nacional.

Figure 3. Representation of various types of social actors among the current members of the REA gobierno (20\%, ministerios y áreas protegidas), los restauradores independientes (10\%) el sector privado $(9 \%$, centros de transferencia tecnológica y empresas), el sector no gubernamental $(3 \%$, organizaciones y fundaciones) y los docentes $(2 \%$, niveles medio y superior). Esta diversidad caracteriza a la fortaleza principal de la REA. Sin embargo, estos actores sociales no están distribuidos de manera homogénea entre los nodos y las ecorregiones (Figura 2). En cierto modo, esta heterogeneidad dificulta la disponibilidad de información, entendida como una evaluación completa de los disturbios principales, las necesidades de restauración desde el punto de vista social y la situación actual de avances en la restauración en todas las ecorregiones del país.

\section{¿Qué falta?}

Las líneas de trabajo necesarias en restauración fueron sistematizadas y agrupadas en los siguientes puntos clave: a) promover una revisión del marco jurídico y de los instrumentos de política pública útiles para fomentar y contextualizar acciones de restauración, así como las herramientas de gestión para su implementación efectiva; b) buscarlosmedios para difundirlainvestigación y la práctica de la restauración; c) promover la participación de la sociedad, el reconocimiento del restaurador y la restauración ecológica como una disciplina, así como aunar esfuerzos con entidades diversas y ámbitos variados (e.g., gubernamentales, no gubernamentales, sociedad en general), incluyendo entidades de gobierno a distintas escalas; d) estimular la implementación de prácticas productivas que minimicen el impacto ambiental y la pérdida de biodiversidad para así reducir las necesidades de restauración ecológica a futuro y; e) definir un marco legal para la red.

\section{¿Hacia dónde vamos?}

Las necesidades globales y regionales para la restauración no sólo se formularon desde los ámbitos académicos en publicaciones científicas, sino que también se cuantificaron y establecieron desde niveles internacionales y compromisos gubernamentales. A modo de ejemplo se pueden citar la meta de Aichi 15 del Convenio sobre la Diversidad Biológica (CBD 2010), el Desafío de Bonn (Bonn Challenge 2014) y la Iniciativa $20 \times 20$, en la cual la Argentina comprometió la restauración de 1 millón de hectáreas (Iniciativa 20×20 2014). Actualmente, en Latinoamérica, sólo Brasil, 
Tabla 1. Líneas potenciales de trabajo para promover acciones de restauración ecológica en el país y consolidar la REA.

Table 1. Potential areas of work to promote ecological restoration actions in Argentina and consolidate the REA.

\begin{tabular}{|c|c|}
\hline \multirow{4}{*}{$\begin{array}{l}\text { Integrar lenguajes, } \\
\text { acordando significados y } \\
\text { definiendo prioridades }\end{array}$} & Acordar e integrar conceptos, criterios y lenguaje sobre la restauración \\
\hline & Establecer prioridades de investigación \\
\hline & Elaborar planes y definición de áreas prioritarias para la restauración \\
\hline & Definir lineamientos y protocolos sobre prácticas y metodología de la restauración \\
\hline $\begin{array}{l}\text { Favorecer el intercambio y } \\
\text { la comunicación horizontal }\end{array}$ & $\begin{array}{l}\text { Intercambio y comunicación horizontal entre individuos, grupos de restauración y } \\
\text { sociedad } \\
\text { Intercambio y comunicación horizontal entre sus nodos regionales }\end{array}$ \\
\hline \multirow{2}{*}{$\begin{array}{l}\text { Sistematizar y compartir } \\
\text { información }\end{array}$} & Integrar información (e.g., bancos de datos, bibliotecas, bibliografía, proyectos, acciones) \\
\hline & $\begin{array}{l}\text { Generar y gestionar una base de datos que contenga la información disponible } \\
\text { Compartir las experiencias exitosas y fallidas (e.g., boletines y publicaciones de amplia } \\
\text { difusión) }\end{array}$ \\
\hline $\begin{array}{l}\text { Capacitar dentro y fuera } \\
\text { de la red }\end{array}$ & $\begin{array}{l}\text { Promover la formación académica orientada a la restauración ecológica en todos los niveles } \\
\text { educativos } \\
\text { Promover la capacitación en nodos regionales (e.g., talleres de actualización, discusión y } \\
\text { comunicación) } \\
\text { Capacitar a distintos actores sociales }\end{array}$ \\
\hline \multirow[t]{4}{*}{ Difundir acciones } & Generar publicaciones con destinatarios y formatos diversos \\
\hline & Organizar eventos (e.g., congresos, reuniones, talleres, capacitación, etc.) \\
\hline & Optimizar, potenciar y mejorar los canales de difusión (e.g., redes sociales) \\
\hline & Divulgar y generar transferencia \\
\hline \multirow{4}{*}{$\begin{array}{l}\text { Promover la } \\
\text { interdisciplinariedad y } \\
\text { la vinculación de actores } \\
\text { sociales }\end{array}$} & $\begin{array}{l}\text { Establecer el vínculo entre quienes generan conocimiento y quienes toman las decisiones, } \\
\text { incorporando el trabajo multidisciplinario e interdisciplinario }\end{array}$ \\
\hline & $\begin{array}{l}\text { Interactuar con los organismos oficiales de ambiente y conservación a nivel nacional, } \\
\text { provincial y local } \\
\text { Integrar disciplinas naturales y sociales en los equipos de restauración }\end{array}$ \\
\hline & Identificar puntos clave de acción, asesoría legal y normativa \\
\hline & Visibilizar los conflictos y necesidades de gestión en políticas públicas de la restauración \\
\hline $\begin{array}{l}\text { Gestionar marco legal y } \\
\text { financiamiento }\end{array}$ & $\begin{array}{l}\text { Promover una participación amplia de actores sociales para la inclusión de la restauración } \\
\text { ecológica como política de Estado } \\
\text { Creación de dependencias responsables de la restauración ecológica a nivel nacional y } \\
\text { local, y generar marcos legales y protocolos de restauración } \\
\text { Generar y difundir modos de financiamiento y ejecución de los planes nacionales e } \\
\text { internacionales de restauración }\end{array}$ \\
\hline
\end{tabular}

Colombia, Ecuador y Guatemala cuentan con planes oficiales de restauración ecológica para ejecutar sus proyectos (Méndez Toribio et al. 2017). Para lograr estas y otras metas es imprescindible tener en cada región del mundo comunidadesderestauradorescomprometidos y capacitados, con experiencia y apoyo institucional (Aronson and Alexander 2013). Desde la perspectiva de la REA, consideramos que para promover y fortalecer la restauración ecológica en el país se necesitan varias líneas de acción y algunas actividades asociadas (Tabla 1). Para consolidar la interacción y la comunicación, $\mathrm{y}$, al mismo tiempo, favorecer la visibilidad, el intercambio y la comunicación horizontal, se plantearon acciones concretas. Algunas de estas acciones son encuentros regionales o nacionales (e.g., talleres y foros) que incluyan a distintos actores sociales (políticos, científicos, técnicos, productores, campesinos) involucrados en la restauración. Estos encuentros ya se realizaron, por ejemplo, en la diagonal árida de la Argentina (Pérez et al. 2013; Carretero and Dalmasso 2015). Futuros talleres, jornadas o foros en diversas regiones servirán para compartir y sistematizar información, acordar significados y un lenguaje común, y definir prioridades tanto en cuestiones geográficas como temáticas. $\mathrm{Al}$ mismo tiempo, la organización de capacitación presencial o a distancia es una herramienta y una necesidad fundamental para formar a los futuros restauradores. La comunicación también se puede fortalecer con el fomento de la interdisciplina y la transdisciplina (ecológica y social), con acciones que permitan vincular a actores sociales y que promuevan una difusión mayor de temas vinculados con la restauración en la sociedad en general.

En la red existe un consenso para promover que se integren a ella todos los individuos $\mathrm{u}$ organizaciones que trabajan en tareas de restauración (e.g., se observa un crecimiento de los grupos que controlan el avance de la flora exótica invasora en diversas regiones del país). Esta integración en la red evitará que dichas acciones se realicen de manera aislada o sin las bases de información necesarias, que lleven a errores o resistencia en la sociedad, 
a la vez que permitan utilizar las lecciones aprendidas.

Consideramos que la consolidación de la REA será un aporte más a los múltiples desafíos de escala, socioeconómicos, institucionales, financieros, de generación de capacidades, indicadores y monitoreo que se requieren resolver. La REA constituye la mayor organización nacional que integra a la comunidad de restauradores con capacidades para aportar a los compromisos de restauración establecidos. Al presente, la red se propone la integración con decisores de la política ambiental en el país y la región, la articulación con grupos de investigación ecológica que cuentan con información de calidad y necesaria para la restauración, y una alta comunicación tanto interna como externa. Como un espacio nacional y regional de convergencia, esta red y sus nodos tienen el potencial para ayudar a identificar los principales desafíos práctico-teóricos de la restauración en la Argentina, y para generar protocolos que ayuden a gestionar acciones de restauración. Finalmente, señalamos que todas las instituciones involucradas en la restauración, integren o no la REA, necesitan incrementar la cantidad y la calidad de sus recursos humanos a fin de formar una masa crítica de profesionales capaces de interpretar los desafíos que enfrenta la restauración en los diversos contextos sociales, culturales y ecológicos del país.

\section{Conclusiones}

La restauración ecológica es una prioridad global y nacional en la que confluyen múltiples actores y disciplinas. Se necesita un espacio en el que converjan las disciplinas involucradas, a fin de contribuir a la construcción social de la importancia y el sentido de la restauración en un escenario de grandes problemas de degradación de los ecosistemas de nuestro país. En este marco se propone un aporte al desarrollo de una restauración ecológica plural y multidisciplinaria, que busque la integración entre teoría y práctica, y la mejor relación entre la sociedad y la naturaleza. Invitamos a la comunidad interesada a participar de la REA y abrimos el debate sobre las líneas de trabajo prioritarias en restauración ecológica en nuestro país.

Agradecimientos. Los autores agradecen a los asistentes del encuentro 2017 de la red REA por su participación y aportes. DP agradece por el financiamiento al I encuentro nacional de restauración ecológica, a la Fundación de la Universidad Nacional del Comahue para el Desarrollo Regional (FUNYDER). PM agradece a FAPESP por una beca postdoctoral (2016/00052-9). DR agradece al

\section{REFERENCIAS}

Aguilar, M., J. Sierra, J. Ramírez, O. Vargas, Z. Calle, W. Vargas, C. Murcia, J. Aronson, and J. Barrera Cataño. 2015. Towards a post-conflict Colombia. Restoring to the future. Restoration Ecology 23:4-6.

Armesto, J. J., S. Bautista, E. Del Val, B. Ferguson, X. García, A. Gaxiola, H. Godinez-Álvarez, G. Gann, F. LópezBarrera, R. Manson, M. Núñez-Ávila, C. Ortiz-Arrona, P. Tognetti, and Guadalupe Williams-Linera. 2007. Towards an ecological restoration network: reversing land degradation in Latin America. Frontiers in Ecology and the Environment 5:W1-W4.

Aronson, J., and S. Alexander. 2013. Ecosystem restoration is now a global priority: Time to roll up our sleeves. Restoration Ecology 21:293-296.

Bonn-Challenge. 2014. URL: www.bonnchallenge.org:

Brancalion, P. H. S., L. C. García, R. Loyola, R. R. Rodrigues, V. D. Pillare, and T. M. Lewinsohn. 2016. A critical analysis of the Native Vegetation Protection Law of Brazil (2012): updates and ongoing initiatives. Natureza e Conservação

14:e1-e16.

CBD (Convention on Biological Diversity). 2010. Strategic plan for biodiversity 2011 - 2020 and the Aichi targets. URL: www.cbd.int/sp/targets.

Carretero, E., and A. D. Dalmasso. 2015. Restauración ecológica en la diagonal árida de la Argentina (2). IADIZA. Mendoza. Pp. 483.

Ceccon, E., J. I. Barrera-Cataño, J. Aronson, and C. Martínez-Garza. 2015. The socioecological complexity of ecological restoration in Mexico. Restoration Ecology 23:331-336

Ceccon, E., and D. R. Pérez (coords.). 2017. Beyond restoration ecology: social perspectives in Latin America and the Caribbean. Vázquez Mazzini Eds. Buenos Aires, Argentina.

Cisneros, L., and E. Cisneros. 2016. Construcción social de la restauración ecológica. En: Ceccon y Pérez (coords.). Más allá de la ecología de la restauración. Vázquez Mazzini Eds. Buenos Aires, Argentina.

Díaz, S., et al. 2018. Assessing nature's contributions to people. Science 359(6373):270-272.

Echeverría, C., C. Smith-Ramírez, J. Aronson, and J. I. Barrera-Cataño. 2015. Good news from Latin America and the Caribbean: national and international restoration networks are moving ahead. Restoration Ecology 23:1-3. 
Gross, M. 2006. Beyond expertise: Ecological science and the making of socially robust restoration strategies. Journal for Nature Conservation 14:172-179.

Higgs, E., D. A Falk, A. Guerrini, M. Hall, J. Harris, R. J. Hobbs, S. T. Jackson, J. M. Rhemtulla, and W. Throop. 2014. The changing role of history in restoration ecology. Frontiers in Ecology and the Environment 12:499-506.

Initiative 20x20. 2014. URL: www.wri.org/our-work/project/initiative-20x20/about-initiative-

20x20\#project-tabs.

Jordan, W. R. 2012. The sunflower forest. Ecological restoration and the new communion with nature. University of California Press Books, Oakland, USA.

Leff, E. 2009. Pensamiento ambiental latinoamericano: patrimonio de un saber para la sustentabilidad. ISEE 6:1-15.

McDonald, T., G. D. Gann, J. Jonson, and K. W. Dixon. 2016. International standards for the practice of ecological restoration - including principles and key concepts. Society for Ecological Restoration, Washington, D.C.

Méndez-Toribio, M., C. Martínez-Garza, E. Ceccon, and M. R. Guariguata. 2017. Planes actuales de restauración ecológica en Latinoamérica: avances y omisiones. Revista de Ciencias Ambientales 51:1-30.

Pérez, D. R., M. E. Rodríguez Araujo, and A. E. Rovere. 2013. Restauración ecológica en la diagonal árida de la Argentina. Vazquez Mazzini Eds. Pp. 515.

Rovere, A. E. 2015. Review of the science and practice of restoration in Argentina: increasing awareness of the discipline. Restoration Ecology 23:508-512.

Soares-Filho, B., R. Rajão, M. Macedo, A. Carneiro, W. Costa, M. Coe, H. Rodrigues, and A. Alencar. 2014. Cracking Brazil's Forest Code. Science 344:363-364.

Society for Ecological Restoration (SER) International, Grupo de trabajo sobre ciencia y políticas. 2004. Principios de SER International sobre la restauración ecológica. URL: www.ser.org y Tucson: Society for Ecological Restoration International.

Suding, K. N. 2011. Toward an era of restoration in ecology: successes, failures, and opportunities ahead. Annual Review of Ecology, Evolution and Systematics 42:465.

Vera, A., D. Tamburini, and F. Barri. 2017. Restauración de áreas desmontadas en infracción. En: La investigación jurídica en políticas públicas ambientales. Parte III: Integración con otras disciplinas. En prensa.

Zuleta, G., A. E. Rovere, D. Pérez, P. I. Campanello, B. Guida Johnson, C. Escartín, A. Dalmasso, D. Renison, N. Ciano, and J. Aronson. 2015. Establishing the ecological restoration network in Argentina: from Rio 1992 to SIACRE 2015. Restoration Ecology 23:95-103. 